\title{
Neurophysiological mechanisms of psychotic symptoms
}

Abstract Psychotic symptoms are supposed to be expression of highest order brain functions such as symbolic thinking, language, planning, empathy or complex emotional reactions. However, due to its historical roots, current psychiatric symptomatology is based on descriptions of disturbed behavior, which refer to metaphysic concepts rather than to brain function. Therefore, modern biological research suffers from an important gap between psychiatric semiology and contemporary knowledge in systems neurophysiology. The authors argue for a redefinition of psychiatric symptoms in a neurobiologically meaningful way. Based on recent empirical studies, this strategy is exemplified for auditory verbal hallucinations and formal thought disorder. In these examples, characteristic psychiatric symptoms can be related to circumscribed structural and functional alterations in the language system, allowing the description of clinical phenomena in terms of neurobiological events. This strategy is also applicable to other psychotic symptoms like emotional dysregulation and catatonia, where disturbances of the functional circuits of mood and motor regulation, respectively, are predicted. This approach to psychiatric symptoms is based on contemporary evidence concerning systems neurophysiology and is expected to provide meaningful and testable hypotheses for future research aimed to a better understanding of the pathogenesis of psychiatric disorders, to more accurate prognosis and to better targeted therapeutic strategies.

Key words psychosis - psychopathology . modularity $\cdot$ hallucinations $\cdot$ thought disorders

W. Strik $(\bowtie) \cdot$ T. Dierks

University Hospital of Psychiatry

University of Bern

Bolligenstrasse 111, Bern 60

3000 Bern, Switzerland

E-Mail: strik@puk.unibe.ch

\section{Introduction}

The physiological basis of high order brain functions such as symbolic thinking, language, planning, empathy or complex emotional reactions is not yet fully understood at the systems level, and, in particular, no single brain region was identified to distinctly represent any of these functions. Accordingly, there is a major conceptual dilemma in modern psychiatry since the foremost paradigm postulates brain dysregulation as the basis of psychiatric symptoms, but there is an evident gap between observed symptoms and systems physiology. The authors will show the need for a reformulation of psychiatric semiology in order to match the behavioral observations with the respective neurophysiological mechanisms.

Historically, the development of current psychopathology was guided by the idea of psychiatry as a science dealing with metaphysical phenomena, arising independently from observable brain processes [14]. This was obviously inspiring the symptom definitions, which were formulated avoiding any reference to known functional neurological circuits. No reference was made, e.g. to speech and language perception physiology although the so-called formal thought disorders are objectively assessed by disturbances of spoken language. Furthermore, fear as a physiological phenomenon with its biologically clearly defined behavioral consequences fight, flight or freezing is not considered in the context of psychosis. Rather, possible cognitive equivalents of these fear-reactions in humans like verbal fight or false accusations are interpreted as distortions of judgment, and avoidance as emotional flattening. To focus the point: in the context of psychosis, aroused emotional states are implicitly supposed to be secondary to the cognitive state and, therefore, psychopathologically negligible as diagnostic signs.

These examples show that current psychiatric semiology contains implicit theoretical assumptions about causality, and that possible alternative hypotheses have not been tested empirically. This is impor- 
tant since evidently every psychopathological scale detects symptoms according to their a priori definition. The consequences for scientific reasoning and for empirical research are far-reaching. If disordered speech is labeled as a thought disorder, and thought is understood as a complex, metaphysical phenomenon, then the way back to brain function is closed. Consequent to this dilemma, modern biological psychiatry rarely refers directly to psychiatric symptoms and the respective psychological functions (e.g. thought, judgment, mood); instead, psychological constructs are applied, which were developed in other contexts like developmental psychology (e.g. theory of mind) or neuropsychology (e.g. working memory, executive function) and can be related to the function of distinct brain circuits. While this is, of course, a legitimate way to overcome the dead end of psychopathology, it is not really a satisfactory one. In fact, the gap between psychiatric symptoms and their underlying brain functions is thus just substituted by a new, unresolved gap between psychiatric symptoms and psychological constructs. E.g. it is not clear how the major symptoms of schizophrenia like hallucinations and thought disorders could be generated by subtle working memory deficits. Such a relationship appears to be easier for the construct of Theory of Mind, where it is hypothesized that a pathological deficit of empathy may lead to delusional false interpretations [1]. This is an interesting approach which, however, tacitly inverts the cognitive paradigm of classical psychopathology which assumes a paranoid delusion to be a primary disorder of judgment and not a secondary, emotion driven cognitive distortion. This is a promising hypothesis, but it should be clear that this explanation implies a new hypothetical bridge from Theory of Mind to the clinical symptoms which needs empirical confirmation. There is a similar problem with respect to the concept of genetic endophenotypes, which allow establishing hypothetical relationships between behavioral phenomena and a genotype. In this case, however, an even larger gap is open between genotype, systems physiology of the brain and behavior.

Although several different research strategies are possible and the verdict about their success or failure is determined by the results, the evident shortcomings of traditional psychopathology due to their implicit, anachronistic theoretical framework appear to impede a meaningful translation of behavior to systems physiology and genetics.

\section{Neuronal networks, distributed circuits, processing domains and behavior}

\section{The neurobiological perspective}

The stratification of complexity in the human brain follows rules that can be understood by their ana- tomical location, e.g. the primary, secondary and association cortex, and the basal ganglia, but only up to the level of organized perception (Gestalt) and movements (Movement Patterns). Higher psychic functions are not located in circumscribed brain regions but are supposed to result from the interaction between distributed brain regions. This means that the emerging phenomena are the equivalent of one or more additional levels of complexity that rely on the functional relationships between brain regions rather than on circumscribed local activity. From a neurobiological perspective one may thus identify the different levels of complexity in the activity of single neurons and neuronal assemblies, local networks and large scale neuronal circuits. Local networks and their activity are not assumed to explain complex cognitive operations. They refer to groups of physically connected neurons which are synchronously active and rather appear to be the carriers of modality specific representations; in the sensory modalities these may be the equivalent of mental images, and in the motor domain, of movement patterns. Mental operations, on the other hand, can be understood as combinations, interactions and sequences of such representations. This is possible due to massive distributed connections provided by the long distance white matter wirings. The highest order of complexity in brain function is thus reflected by distributed neuronal circuits, which are not defined by simultaneous activation but rather by interactions between brain regions, the temporal dynamics of the system and the output at the behavioral level.

\section{The psychological perspective}

Until today, the psychological perspective on brain functioning is determined by the concept of information processing. In its first formulations, the process was understood as a single channel with limited capacity. It could be regularly fed or overloaded, and psychological experiments were designed in order to segregate information processing into distinct steps. It became evident, however, that a single, serial process is not sufficient to explain the complexity of human behavior. An early formulation of the parallel processing theory implied splitting of the information stream, and the processing of distinct information packages in specialized subroutines, which are supposed to work simultaneously and join together to form the final behavioral output [5]. A limitation of the original formulation of parallel processing for the explanation of complex brain functions is the assumption of highly specialized, encapsulated subroutines excluding top down inputs.

A modern formulation of the parallel processing theory consists in massive modularity. It complies with the evidence of top down, i.e. state dependent modulations of cognitive processes. Subroutines are 
organized in functional units, which can be identified as specialized neuronal circuits. These circuits are modulated by the general systems state which means, in psychological terms, by memories and intentions. Further implications include the key-lock principle, which makes a distinct upstream routing device to screen, classify and assign information packages superfluous. Rather, the large-scale availability of information in a massively networked system allows each module to respond only to its specific domain, which is defined by the format rather than by the content of the information.

Massive modularity allows clarifying some crucial issues around the function of specialized brain circuits. In this view, e.g., the question of whether the fusiform face area is specialized to human faces (e.g. [11]) is ill targeted. Instead, the region is specialized to a particular visual pattern; let's say ovals with a structured surface. This specific format of the information is the key for being processed by the module; its content and the meaning, in this example a particular face, is not the prerequisite of the input, but the output, i.e. the result of the module's processing. Similar forms are equally treated as a proper input and duly processed and distinguished, like animal faces, car fronts or the moon. Correspondingly, the domain of the language system is not language itself, but acoustic packages with the format of syllables. Words, sentences and meanings are, again, not the domain of the system but its output. The concept of massive modularity very much approximates the idea of a possible translation between the psychological and the neurobiological level of observation. It further provides the domains as a useful conceptual distinction between format and content of the information as the key to enter the processing of a particular module. Finally it is compatible with the notion of the brain as a self organizing system making the existence of an intelligent upstream router superfluous [2].

\section{Consequences for a biological psychopathology}

To better understand how psychiatric signs and symptoms can be translated into brain dysfunctions, it appears necessary to rely on normal brain functions which can be related to both, psychiatric symptomatology and distributed neuronal circuits with distinct tasks and output. As shown above, psychological modules can be understood as the equivalent of distributed circuits, and one key is to define the domain of specialization based on the format of the information rather than on its content. This prerequisite is not fulfilled by the disturbances of thought, judgment or mood to which psychopathology traditionally refers. They are defined based on content and meaning. Psychological modules like working memory or attention, on the other hand, are well studied in terms of brain circuitry. However, their relationship to the specific signs and symptoms of psychosis is unclear.
The intriguing hyperarousal/information overflow theory exemplifies the dilemma, since the respective clinical syndrome is rarely and, at most, only transitorily seen in psychosis. It is highly unspecific and cannot be easily linked to the specific pathophysiology of psychosis.

To recruit the modern concepts of brain modules and circuits for the neurobiological understanding of psychotic symptoms, possible candidates for the generation of psychotic symptoms must be concretely nominated, however [6]. For this purpose it is helpful to identify a critical communication breakdown as a common denominator of the clinical condition of psychosis, and then to look at the major domains of human communication. Three important communication domains can be attributed without difficulty to specialized brain circuits: The left hemispheric language system for verbal, the limbic system for emotional and the antero-medial motor loop for the psycho-motor behavior; the latter being crucial for non-verbal communication, e.g. of sympathy or intentions. A respective formulation of psychopathology aimed to match clinical phenomena to these circuits has been developed recently and is currently being validated by our group. While the clinical expressions of dysregulations of the limbic loop are not trivial to identify, the disturbances of psychomotor behavior and of language are easier to understand. Research is still in its beginnings regarding such a strategy. However, based on symptom oriented investigations, several interesting results are already available with regard to the language system in schizophrenia. In the following part we will review findings regarding those psychotic symptoms, which can be directly attributed to the language circuitry, namely the auditory hallucinations, and formal thought disorders.

\section{The pathophysiology of auditory hallucinations}

Hallucinations as they typically occur in schizophrenia commonly contain spoken language in the form of words or entire sentences. Their relationship to the language system of the human brain is therefore evident. However, until recently the involved brain regions and the dynamics of their emergence were unclear.

One distinctive feature of hallucinations is their physical intensity similar to a real perception, and the subjective sense of being externally generated [3]. In a fMRI study in hallucinating patients with schizophrenia Dierks et al. [4] showed, that during auditory hallucinations besides motor speech (Broca's) area the primary auditory cortex (Heschl's gyrus) in the language dominant hemisphere was activated. The coactivation of the left temporal primary auditory cortex can be thus understood as an abnormal excitatory 
phenomenon which is related to the subjective experience of a real acoustic stimulus. As such, this region adds a vivid physical quality to a conscious event in the language domain. In other words, a conscious verbal content may be identified as internally - self or externally generated based on the simultaneous neural activity of the primary cortex [16].

The intrahemispheric connection between the frontal and the temporal language related regions is provided by the arcuate fascicle. The lateral part of this fiber bundle connects the inferior prefrontal lobe with temporoparietal areas at the end of the sylvic fissure and the superior temporal lobe. When comparing two groups of patients with schizophrenia, one with patients who either hallucinated frequently and another who had never experienced hallucinations, with normal controls, the group of hallucinating patients had a specific increase of directionality (MRI fractional anisotropy) of white matter tracts in the lateral part of the arcuate fascicle pronounced in the left hemisphere. This effect was present compared to both, normal controls and nonhallucinating patients with schizophrenia [10]. This indicates a structural increase of the fronto-temporal connections of the language circuit which is consistent with a functional dysregulation between speech generating frontal and speech perceiving temporal regions.

It was hypothesized that a higher directionality of the arcuate fascicle facilitates the pathological retrograde co-activation of the primary auditory cortex as part of the described dysfunctional systems state representing the auditory hallucination [15]. However, it remains open, whether this finding is the primary, neurodevelopmental cause of the suspected excitatory derailment of the temporal lobe or whether it is a phenomenon of neural plasticity following a chronic state of hyperactivity of the language circuit.

Early evidence that auditory hallucinations are connected to anatomical structures and pathways related to the language system came from the fact that patients with lesions in these regions e.g. due to tumors, infarctions, hemorrhages or epileptic foci, not only suffered from speech disturbances, but eventually also from auditory hallucinations. Support for the hypothesis that auditory hallucinations are misinterpreted inner speech and closely related to physiological language circuits recently came from a case of a 63 year old woman with a vascular and traumatic injury of the left frontal and temporal language regions described recently by Hubl et al. [9]. This patient was dysphasic after the acute lesions. During recovery, she developed epileptic spikes in the left superior temporal lobe, and auditory verbal hallucinations. While she recognized external speech e.g. of the nursing staff as normal, she was able to recognize that voices she hallucinated had the same dysphasic speech difficulties like herself. Taken together these studies provide evidence that different aspects of the psychiatric psychopathological symptom of hallucinations are related to specific parts of the language processing and generation system bridging the gap between psychopathology and brain function.

\section{Formal thought disorders and the language circuit}

Another group of symptoms which can be related to language dysfunctions is formal thought disorder. It is still a heterogeneous group of phenomena which, in linguistic terms, can be defined as errors in logical sequencing and grammar, loosened or restricted associations or as an increased or decreased flow and quantity of speech. Several studies have demonstrated functional and structural deficits in the left superior temporal regions particularly consistent when related to formal thought disorders [13]. These results are consistent with studies demonstrating receptive language deficits in schizophrenia. Examples of functional changes related to language processing are the reduction of the P300 amplitude at left temporal regions which was correlated with the performance in a verbal memory task $[7,17]$, and the reduction of the neuronal (fMRI) response during the generation of speech which was attenuated with increasing formal disorders of language [12].

These studies do not differentiate between activation deficits and abnormalities of spontaneous activity. This is due to the method which is only able to detect difference values between basal activity and activity increase after stimulation. Thus, the alternative explanation is possible, that the relative activation related to a specific brain function is reduced due to a ceiling effect in an intrinsically hyperactive region. Therefore, to obtain a more complete picture of the neuronal activity during rest and functional activation, absolute perfusion measures are required.

In a recent study, Horn et al. [8] investigated the relationship between grey matter volume, absolute blood perfusion in language related brain areas and formal thought disorders in a group of patients with schizophrenia and healthy controls. In accordance with previous studies, in the left temporal lobe a region with reduced gray matter volume was found. Further, there were interesting correlations of formal thought disorders with grey matter volume on one hand, and absolute perfusion on the other. In particular, formal thought disorders were associated with reduced grey matter volume and increased perfusion in left superior posterior temporal regions, and with increased perfusion but not with grey matter volume in left infero-prefrontal areas. Together with previous activation studies these results are interpreted as an indication that formal thought disorders are characterized by a structurally impaired Wernicke's region along with a hyperexcitation of the left hemispheric language system. 


\section{Conclusion}

The example of the language circuitry shows that an empirically based translational approach from systems physiology to psychopathology is possible. A similar approach can be, in principle applied to the other major communication domains, namely affectivity and motor behavior. While motor behavior appears to be easily accessible to such a conceptualization, affectivity is less trivial since it is intricate with thought, judgment and behavior in a largely unknown extent and with unclear causalities. The effort, however, to better identify specific symptoms which can be translated to the activity of brain circuitries appears to be worthwhile since it may represent an important step towards the understanding of the nature of psychoses.

Disclosure No conflicts of interest.

\section{References}

1. Andreasen NC, Calage CA, O'Leary DS (2008) Theory of mind and schizophrenia: a positron emission tomography study of medication-free patients. Schizophr Bull

2. Barrett HC, Kurzban R (2006) Modularity in cognition: framing the debate. Psychol Rev 113:628-647

3. David AS (2004) The cognitive neuropsychiatry of auditory verbal hallucinations: an overview. Cognit Neuropsychiatry 9:107-123

4. Dierks T, Linden DEJ, Jandl M, Formisano E, Goebel R, Lanfermann H, Singer W (1999) Activation of Heschl's Gyrus during auditory hallucinations. Neuron 22:615-621
5. Fodor J (1983) The modularity of mind. Cambridge

6. Gaebel W, Zielasek J (2008) The DSM-V initiative "deconstructing psychosis" in the context of Kraepelin's concept on nosology. Eur Arch Psychiatry Clin Neurosci 258(Suppl 2):4147

7. Heidrich A, Strik WK (1997) Auditory P300 topography and neuropsychological test performance: evidence for left hemispheric dysfunction in schizophrenia. Biol Psychiatry 41:327335

8. Horn H, Federspiel A, Wirth M, Müller TJ, Wiest R, Wang JJ, Strik W (2008) Structural and metabolic changes in language areas linked to formal thought disorder. Br J Psychiatry (in press)

9. Hubl D, Hauf M, van Swam C, Muri R, Dierks T, Strik W (2007) Hearing dysphasic voices. Lancet 370:538-538

10. Hubl D, Koenig T, Strik W, Federspiel A, Kreis R, Boesch C, Maier SE, Schroth G, Lovblad K, Dierks T (2004) Pathways that make voices-white matter changes in auditory hallucinations. Arch Gen Psychiatry 61:658-668

11. Kanwisher N, Yovel G (2006) The fusiform face area: a cortical region specialized for the perception of faces. Philos Trans $R$ Soc Lond B Biol Sci 361:2109-2128

12. Kircher TT, Liddle PF, Brammer MJ, Williams SC, Murray RM, McGuire PK (2001) Neural correlates of formal thought disorder in schizophrenia: preliminary findings from a functional magnetic resonance imaging study. Arch Gen Psychiatry 58:769-774

13. Kircher TT, Thienel R (2005) Functional brain imaging of symptoms and cognition in schizophrenia. Prog Brain Res 150:299-308

14. Schneider K (1919) Reine psychiatrie, symptomatische psychiatrie und neurologie. Zeitschr $\mathrm{f} d$ ges Neur u Psych 49

15. Strik W, Dierks T (2005) How modern neurophysiology can help to understand schizophrenia. Swiss Arch Neurol Psychiatry $155: 368-374$

16. Strik W, Dierks T, Hubl D, Horn H (2008) Hallucinations, thought disorders, and the language domain in schizophrenia. Clin EEG Neurosci 39:91-94

17. Strik WK, Dierks T, Maurer K (1993) Amplitudes of auditory P300 in remitted and residual schizophrenics-correlations with clinical-features. Neuropsychobiology 27:54-60 